\title{
Variational localized-site cluster expansions. X. Dimerization in linear Heisenberg chains
}

\author{
D. J. Klein* and M. A. García-Bach ${ }^{\dagger}$ \\ Department of Physics, Austin, Texas 78712
}

(Received 14 July 1977)

\begin{abstract}
Ground-state instability to bond alternation in long linear chains is considered from the point of view of valence-bond (VB) theory. This instability is viewed as the consequence of a long-range order (LRO) which is expected if the ground state is reasonably described in terms of the Kekule states (with nearest-neighbor singlet pairing). It is argued that the bond alternation and associated LRO predicted by this simple VB picture is retained for certain linear Heisenberg models; many-body VB calculations on spin $s=1 / 2$ and $s=1$ chains are carried out in a test of this argument.
\end{abstract}

\section{INTRODUCTION}

There is much interest concerning dimerization in linear-chain systems. In fact, generally in an infinite-chain system, for which a simple halffilled-band picture applies, bond alternation is favored; at high temperatures such a chain might ${ }^{2}$ be uniform, in which case a "Peierls" transition to a dimerized (or alternating) structure occurs at lower temperatures. Related transitions have been proposed, ${ }^{3}$ studied, ${ }^{4-6}$ and observed ${ }^{6}$ for linear arrays of doublet transition-metal ions described by the nearest-neighbor $s=\frac{1}{2}$ Heisenberg model.

Peierls transitions have been observed ${ }^{7}$ and studied $^{8}$ for a variety of tetracyanoquinodimethane and Krogman salts. Bond-alternation studies ${ }^{9,10}$ for linear polyenes have also been popular. A common feature of almost all of this work is that HartreeFock solutions are employed.

Here the dimerization problem for linear chains is approached in another way, which is not predicated upon an independent-electron picture. Although this new valence-bond (VB) cluster approach should be applicable to many of the abovementioned cases, here we shall study just Heisenberg models, typically of the form

$$
H=J \sum_{i=1}^{2 n} \overrightarrow{\mathrm{s}}_{i} \cdot \overrightarrow{\mathrm{s}}_{i+1}+\delta J \sum_{i=1}^{2 n}(-1)^{i} \overrightarrow{\mathrm{s}}_{i} \cdot \overrightarrow{\mathrm{s}}_{i+1},
$$

where the exchange parameters are antiferromagnetically signed $J(1 \pm \delta)>0$, the (even) chain length will be taken to infinity, and $\delta$ measures the extent of dimerization. Clearly, if the ground-state energy to (1.1) is linear in $\delta$ and the other (Coulombic) restoring forces are harmonic, then dimerization should occur, at least at absolute zero.

The general qualitative VB argument which can indicate dimerization in a very long even cyclic chain (of equivalent sites) is rather simple. ${ }^{11}$ Indeed, the two singlet Kekule structures, which one expects to describe the ground state, are essentially not only orthogonal but also noninteracting (each to order $\sim 2^{-n}$ ). Thus, when the sites are equally spaced there is an essential degeneracy, with each Kekule structure responding essentially independently to dimerizing perturbations. But a single Kekulé structure responds linearly to dimerizing perturbations, because of the alternating structure inherent in a single Kekule structure, and one expects bond alternation.

Of course this is only a qualitative picture (much as the Neél state provides only a qualitative picture of antiferromagnetic instabilities for lattices of paramagnetic ions). Whether in fact this simple picture actually suggests the correct behavior depends upon the nature of the particular linear model under consideration and upon the effect of other relevant VB structures. Indeed, under certain conditions we show that this dimerization conclusion is not spoiled by inclusion of other VB structures; moreover, we argue that these conditions are valid for Heisenberg models as in (1.1) when the site spin $s$ is such that $2 s$ is an odd integer.

In Sec. II Kekule structures are further considered, and it is pointed out that they exhibit longrange order (LRO), as measured by the LRO parameter $\rho$,

$$
\begin{aligned}
\rho^{2} \equiv \lim _{\alpha \rightarrow \infty}(-1)^{q} \lim _{n \rightarrow \infty} & \left\langle\left(\overrightarrow{\mathrm{s}}_{i} \cdot \overrightarrow{\mathrm{s}}_{i+1}-\left\langle\overrightarrow{\mathrm{s}}_{i} \cdot \overrightarrow{\mathrm{s}}_{i+1}\right\rangle\right)\right. \\
& \left.\times\left(\overrightarrow{\mathrm{s}}_{i+q} \cdot \overrightarrow{\mathrm{s}}_{i+q+1}-\left\langle\overrightarrow{\mathrm{s}}_{i+q} \cdot \overrightarrow{\mathrm{s}}_{i+q+1}\right)\right)\right\rangle,
\end{aligned}
$$

with the expectation values in general being over a state (or ensemble) of interest. Sections III and IV present general arguments that this LRO persists in the ground state of certain Heisenberg models. Section $V$ points out that the existence of this type of LRO indicates bond alternation. For the $s=\frac{1}{2}$ and $s=1$ chains Secs. VI and VII give numerical results, as obtained by the VB cluster approach of the preceeding paper. ${ }^{12}$ Finally our $s=\frac{1}{2}$ results are argued to be more reliable than earlier results..$^{4-6,13,14}$ 


\section{KEKULÉ STATES AND LRO}

First we consider the linear spin $s$ Heisenberg model of (1.1), for which the ground state is known to $\mathrm{be}^{15}$ an overall singlet. A singlet pairing of two nearest-neighbor sites is energetically favored over the triplet pairing, so we consider the simplest (overall singlet symmetry adapted) VB description of the ground state for a cyclic uniform $(\delta=0)$ chain to be

$$
|\psi\rangle \equiv \frac{1}{\sqrt{2}}(|a\rangle+|b\rangle)
$$

where $|a\rangle$ and $|b\rangle$ represent the two Kekule structures

$$
\begin{aligned}
& |a\rangle \equiv 2^{-n / 2} \prod_{i=1}^{n}[2 i-1,2 i], \\
& |b\rangle \equiv 2^{-n / 2} \prod_{i=1}^{n}[2 i+1,2 i],
\end{aligned}
$$

and $[i, j]$ represents the singlet pairing function for sites $i$ and $j$,

$$
\cdot[i, j] \equiv \sum_{m=-s}^{+s}(-1)^{s-m} \varphi_{i}^{s m} \varphi_{j}^{s \bar{m}}
$$

with $\varphi_{i}^{s m}$ the spin function for site $i$ having $z$ component of spin $m$. Here not only are $|a\rangle$ and $|b\rangle$ normalized but also $|\psi\rangle$ is, at least asymptotically, since (using Pauling's island counting technique ${ }^{12}$ )

$$
\langle a \mid b\rangle=(2 s+1)^{-n}(2 s+1) \sim(2 s+1)^{-n} .
$$

Similarly we see that

$$
\left\langle a\left|\prod_{i, j}^{l} \vec{s}_{i}: \vec{s}_{j}\right| b\right\rangle \sim(2 s+1)^{-n}
$$

since the finite number of spin operators in the $l$ fold product $\Pi_{i, j}^{l} \vec{s}_{i} \cdot \vec{s}_{j}$ could introduce no more than a finite number of islands. Then,

$$
\begin{aligned}
\left\langle\psi\left|\prod_{i, j}^{l} \overrightarrow{\mathrm{s}}_{i} \cdot \overrightarrow{\mathrm{s}}_{j}\right| \psi\right\rangle \cong & \frac{1}{2}\left\langle a\left|\prod_{i, j}^{l} \overrightarrow{\mathrm{s}}_{i} \cdot \overrightarrow{\mathrm{s}}_{j}\right| a\right\rangle \\
& +\frac{1}{2}\left\langle b\left|\prod_{i, j}^{l} \overrightarrow{\mathrm{s}}_{i} \cdot \overrightarrow{\mathrm{s}}_{j}\right| b\right\rangle .
\end{aligned}
$$

Further,

$$
\begin{aligned}
& \left\langle a\left|\left(\prod_{i, j}^{l} \overrightarrow{\mathrm{s}}_{i} \cdot \overrightarrow{\mathrm{s}}_{j}\right)\left(\prod_{i, j^{\prime}}^{l \cdot} \overrightarrow{\mathrm{s}}_{i}, \cdot \overrightarrow{\mathrm{s}}_{j^{\cdot}}\right)\right| a\right\rangle \\
& \cong\left\langle a\left|\prod_{i, j}^{l} \overrightarrow{\mathrm{s}}_{i} \cdot \overrightarrow{\mathrm{s}}_{j}\right| a\right\rangle\left\langle a\left|\prod_{t^{\prime}, j^{\prime}}^{l^{\circ}} \overrightarrow{\mathrm{s}}_{i^{\prime}} \cdot \overrightarrow{\mathrm{s}}_{j^{\prime}} \cdot\right| a\right\rangle, \\
& \left\langle b\left|\left(\prod_{i, j}^{l} \overrightarrow{\mathbf{s}}_{i} \cdot \overrightarrow{\mathrm{s}}_{j}\right)\left(\prod_{i^{\prime}, j^{\prime}}^{l^{\circ}} \overrightarrow{\mathrm{s}}_{i^{\prime}} \cdot \overrightarrow{\mathrm{s}}_{j^{\prime}}\right)\right| b\right\rangle \\
& \cong\left\langle b\left|\prod_{i, j}^{l} \overrightarrow{\mathbf{s}}_{i} \cdot \overrightarrow{\mathbf{s}}_{j}\right| b\right\rangle\left\langle b\left|\prod_{i, j}^{l \cdot} \cdot \overrightarrow{\mathbf{s}}_{i}, \cdot \overrightarrow{\mathbf{s}}_{j} \cdot\right| b\right\rangle,
\end{aligned}
$$

for two separate sets of site indices (involved in $\Pi_{i, j}^{l}$ and $\left.\Pi_{i^{\prime}, j^{\prime}}^{l,}\right)$ with all the sites in one set distant from those in the other.

Of key significance in our discussion is the value of the LRO parameter $\rho$ of (1.2), so that it is of interest to evaluate $\rho$ for the simple superposition $|\psi\rangle$ of the two Kekule structures. Thus, noting the translational symmetry of $|\psi\rangle$ and the result of (2.6), we have

$\rho^{2}=\lim _{q \rightarrow \infty} \frac{1}{2}(-1)^{q-1} \lim _{n \rightarrow \infty}\left[\left\langle a\left|\left(\overrightarrow{\mathrm{s}}_{1} \cdot \overrightarrow{\mathrm{s}}_{2}-\left\langle\overrightarrow{\mathrm{s}}_{1} \cdot \overrightarrow{\mathrm{s}}_{2}\right\rangle\right)\left(\overrightarrow{\mathrm{s}}_{q} \cdot \overrightarrow{\mathrm{s}}_{q+1}-\left\langle\overrightarrow{\mathrm{s}}_{q} \cdot \overrightarrow{\mathrm{s}}_{q+1}\right\rangle\right)\right| a\right\rangle+\left\langle b\left|\left(\overrightarrow{\mathrm{s}}_{1} \cdot \overrightarrow{\mathrm{s}}_{2}-\left\langle\overrightarrow{\mathrm{s}}_{1} \cdot \overrightarrow{\mathrm{s}}_{2}\right\rangle\right)\left(\overrightarrow{\mathrm{s}}_{q} \cdot \overrightarrow{\mathrm{s}}_{q+1}-\left\langle\overrightarrow{\mathrm{s}}_{q} \cdot \overrightarrow{\mathrm{s}}_{q+1}\right\rangle\right)\right| b\right\rangle\right]$.

Now, using (2.7) and then (2.6) again, we obtain

$$
\begin{aligned}
\rho^{2}=\lim _{a \rightarrow \infty} \frac{(-1)^{q-1}}{4} \lim _{n \rightarrow \infty} & \left(\left\langle a\left|\overrightarrow{\mathbf{s}}_{1} \cdot \overrightarrow{\mathrm{s}}_{2}\right| a\right\rangle-\left\langle b\left|\overrightarrow{\mathrm{s}}_{1} \cdot \overrightarrow{\mathrm{s}}_{2}\right| b\right\rangle\right) \\
& \times\left(\left\langle a\left|\overrightarrow{\mathbf{s}}_{q} \cdot \overrightarrow{\mathbf{s}}_{q+1}\right| a\right\rangle-\left\langle b\left|\overrightarrow{\mathbf{s}}_{q} \cdot \overrightarrow{\mathrm{s}}_{q+1}\right| b\right\rangle\right) .
\end{aligned}
$$

Finally, noting that the translation of $|a\rangle$ one step yields just $-|b\rangle$, we have

$$
\rho^{2}=\frac{1}{4} \lim _{n \rightarrow \infty}\left(\left\langle a\left|\overrightarrow{\mathbf{s}}_{1} \cdot \overrightarrow{\mathrm{s}}_{2}\right| a\right\rangle-\left\langle b\left|\overrightarrow{\mathrm{s}}_{1} \cdot \overrightarrow{\mathrm{s}}_{2}\right| b\right\rangle\right)^{2},
$$

so that

$$
\rho=\frac{1}{2}\left|\left\langle a\left|\overrightarrow{\mathbf{s}}_{1} \cdot \overrightarrow{\mathrm{s}}_{2}-\overrightarrow{\mathbf{s}}_{2} \cdot \overrightarrow{\mathrm{s}}_{\mathbf{3}}\right| a\right\rangle\right| .
$$

For the Kekule states numerical evaluation is very simple at this stage and gives $\rho=\frac{1}{2} s(s+1)$.

\section{LRO FOR $s=\frac{1}{2}$ AND $s=1$ CHAINS}

We wish to argue that LRO, of the type in (1.2), will persist for $s=\frac{1}{2}$ and $s=1$ linear Heisenberg models even when more accurate (singlet VB) ground-state wave functions are constructed. In these cases we can ${ }^{12}$ apply spin-pairing schemes, wherein any singlet ket is a linear combination of VB structure kets with each site involved in a singlet coupled pair, as in (2.3). The main assumption of our development here will be that in the ground-state spin pairing between a pair of 
sites should only be important when the two sites are not very distant from one another. This main assumption can be argued for $s=\frac{1}{2}$ to be reasonable in a chemist's language because it is generally conceived that bonds between distant sites are unimportant. In a physicist's language this main assumption might be argued to be reasonable, since otherwise there would tend to be long-range correlations (and order) of the type in the Neél state. Also, we could argue its reasonableness if we believe the Kekulé state to provide a good zeroorder picture, and then note that the nearness of spin pairing tends to be preserved on applying the Hamiltonian $H$.

Before using our main assumption we consider a classification of VB structures for a very large cyclic chain of sites. The classification proceeds through a consideration of two conditions: (i) site $j$ is bonded (i.e., spin paired) to the nearestneighbor site $j+1$; and (ii) there is no bonding "through" site $j$, i.e., there is no site near to and the right of $j$. Now, we classify a VB structure as:

(a) type $A$, if whenever (i) and (ii) hold, site $j$ is odd; (b) type $B$, if whenever (i) and (ii) hold site $j$ is even; (c) type $C$, if it is not of type $A$ or $B$. Clearly, the Kekule structures $|a\rangle$ and $|b\rangle$ are of types $A$ and $B$, respectively. In fact, all the physically reasonable $\mathrm{VB}$ structures, i.e., those satisfying our main assumption concerning the unimportance of long bonds, are of type $A$ or type $B$. (This is easily seen, for if we have two nearest-neighbor pairs of spin-paired sites, say $j \sim j+1$ and $j^{\prime} \sim j^{\prime}+1$, then we see that the only way to obtain an odd number of sites between these two pairs would be to have at least one bond through one of these pairs.)

Thus, given our main assumption we conclude the $\delta=0$ ground-state wave function can be written (at least asymptotically), in the form

$$
|\Psi\rangle=1 / \sqrt{2}(|A\rangle+|B\rangle)
$$

where $|A\rangle$ and $|B\rangle$ are composed from VB structures of types $A$ and $B$, respectively. Much as for Kekulé states we expect kets of type $A$ and $B$ to be asymptotically orthogonal and noninteracting. ${ }^{16}$ Consequently, the development involving Eqs. (2.6)-(2.12) still holds, and the LRO parameter for $|\Psi\rangle$ is

$$
\rho=\frac{1}{2}\left|\left\langle A\left|\left(\overrightarrow{\mathrm{s}}_{1} \cdot \overrightarrow{\mathrm{s}}_{2}-\overrightarrow{\mathrm{s}}_{2} \cdot \overrightarrow{\mathrm{s}}_{3}\right)\right| A\right\rangle\right| .
$$

Thus $\rho$ is expected to be nonzero.

\section{LRO FOR $2 s$ AN ODD INTEGER}

We can make a slightly modified argument leading to the prediction of the same type of LRO whenever $2 s$ is an odd integer. For these cases the ground state is ${ }^{15}$ still an overall singlet, even though spin-pairing schemes no longer ${ }^{12}$ generally apply. Our modified main assumption is that in the ground-state local-singlet spin couplings for a set of sites are important only when the sites are all near to one another. This assumption can be viewed as saying that the singlet VB cluster expansion for the ground state converges rapidly. Some of the same rationales as mentioned in Sec. III can also be invoked here to argue for the present assumption.

Now, we note that a set of spin-s sites, with $2 s$ an odd integer, can only be coupled to a singlet when there is an even number of sites in this set. Consequently, only even numbered site excitations occur in the VB cluster ansatz, and a classification into $A, B$, and $C$ types of spin couplings occurs, much as in Sec. III. The main assumption again precludes type- $C$ spin couplings, and LRO results.

This argument fails for integer spin sites because, for instance, three sites can be coupled to a singlet, and three-site terms could be important in the VB cluster ansatz. Since such odd numbered excitations can occur, two pair excitations (wherein nearest-neighbor pairs of sites are singlet paired) can have an odd number of intervening sites coupled to a singlet, and the main assumption fails to preclude spin couplings of type C. For these spins we then expect no LRO.

\section{LRO AND DIMERIZATION}

Once we have admitted the type of LRO described in Secs. II and III the tendency for bond alternation readily follows. Clearly both the $(+)$ and (-) linear combinations of $|A\rangle$ and $|B\rangle$ exhibit the full translational symmetry expected of eigenkets to the $\delta=0$ model and both are asymptotically (i.e., for very large $n$ ) degenerate for $\delta=0$. For $\delta$ deviating just very slightly from zero, one or the other of the two essentially noninteracting kets $|A\rangle$ or $|B\rangle$ will be lower in energy and will essentially completely dominate in the wave function. Hence, the rate of change of the ground state energy $E_{0}$ with respect to $\delta$ will be given by

$$
\begin{aligned}
\left(\frac{\partial}{\partial \delta} \frac{E_{6}}{J N}\right)_{6 \rightarrow 0-} & =-\left\langle A\left|\left(\frac{\partial H}{\partial \delta}\right)_{6=0}\right| A\right\rangle \frac{1}{J N} \\
& =-\left\langle A\left|\frac{1}{N} \sum_{i=1}^{2 n}(-1)^{i} \overrightarrow{\mathrm{s}}_{i} \cdot \overrightarrow{\mathrm{s}}_{i+1}\right| A\right\rangle \\
& =\frac{1}{2}\left\langle A\left|\left(\overrightarrow{\mathrm{s}}_{1} \cdot \overrightarrow{\mathrm{s}}_{2}-\overrightarrow{\mathrm{s}}_{2} \cdot \overrightarrow{\mathrm{s}}_{3}\right)\right| A\right\rangle=+\rho .
\end{aligned}
$$

Thus the presence of LRO of the type defined in (1.2) leads to a nonvanishing linear ground state 
response to the dimerizing $\delta$ perturbation, and the uniform chain is not energetically favored.

\section{NUMERICAL RESULTS FOR $s=\frac{1}{2}$ CHAINS}

We use our ${ }^{12}$ many-body VB cluster ansatz to make more accurate numerical estimates of the LRO parameter $\rho$ for $s=\frac{1}{2}$ Heisenberg models. In restricting the cluster expansion to six-site terms the wave function ansatz takes the general form

$$
\begin{aligned}
& |\Psi\rangle \equiv \mathcal{u}_{s}\left(e^{T+}\right)|\Phi\rangle \\
& T^{+} \equiv \sum_{i=1}^{2 n}\left(T_{i i+1}^{+}+T_{i i+1 i+2 i+3}^{+}+T_{i i+1 i+2 i+3 i+4 i+5}^{+}\right),
\end{aligned}
$$

where $^{12}$ the script $\mathfrak{u}$ indicates that only unlinked terms are to be retained and the subscript $s$ on $u$ indicates an excitation is to occur on every site. The excitation operators are chosen to give rise to excitations

$$
\begin{aligned}
& T_{i i+1}^{+}\left|g_{i} g_{i+1}\right\rangle=(-1)^{i} x_{i}[i, i+1], \\
& T_{i i+1 i+2 i+3}^{+}\left|g_{i} g_{i+1} g_{i+2} g_{i+3}\right\rangle=y_{i}[i, i+3][i+2, i+1], \\
& T_{i i+1 i+2 i+3 i+4 i+5}^{+}\left|g_{i} g_{i+1} g_{i+2} g_{i+3} g_{i+4} g_{i+5}\right\rangle \\
& \quad=(-1)^{i} z_{i}[i, i+5][i+2, i+1][i+4, i+3],
\end{aligned}
$$

where $g_{j}$ indicates some site ground state for site $j$. These excitations of (6.2) are associated with the Rumer diagrams of Fig. 1. The four-site excitations giving rise to $[i, i+1][i+2, i+3]$ have been neglected here, since preliminary computations indicated they were unimportant. Other more complicated six and more-site excitations were neglected to keep the computations more tractable. For $\delta \neq 0$ the symmetry of the Hamiltonian (1.1) causes an alternation in the variational parameters $x_{i}, y_{i}$, and $z_{i}$. In fact we realize that only states of type $A$ (if $\delta<0$ ) or of type $B$ (if $\delta>0$ ) will survive, and we may choose $x_{i}=y_{i}=z_{i}=0$ for either all even $i$ (if $\delta \leqslant 0$ ) or all odd $i$ (if $\delta \geqslant 0$ ). The remaining nonzero parameters we now simply denote by $x, y, z$. In Appendix A we use the matrix element evaluation techniques of earlier paper $\mathrm{s}^{17,18}$ to obtain an energy expression

$$
\frac{E_{6}}{J N}=\frac{1+|\delta|}{2} \cdot \frac{\left\langle\Psi\left|\overrightarrow{\mathrm{s}}_{1} \cdot \overrightarrow{\mathrm{s}}_{2}\right| \Psi\right\rangle}{\langle\Psi \mid \Psi\rangle}+\frac{1-|\delta|}{2} \frac{\left\langle\Psi\left|\overrightarrow{\mathrm{s}}_{2} \cdot \overrightarrow{\mathrm{s}}_{3}\right| \Psi\right\rangle}{\langle\Psi \mid \Psi\rangle},
$$

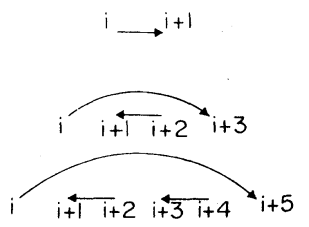

FIG. 1. Diagrams representing the spin pairings occurring in the two-, four-, and six-site excitation operators of (6.2).

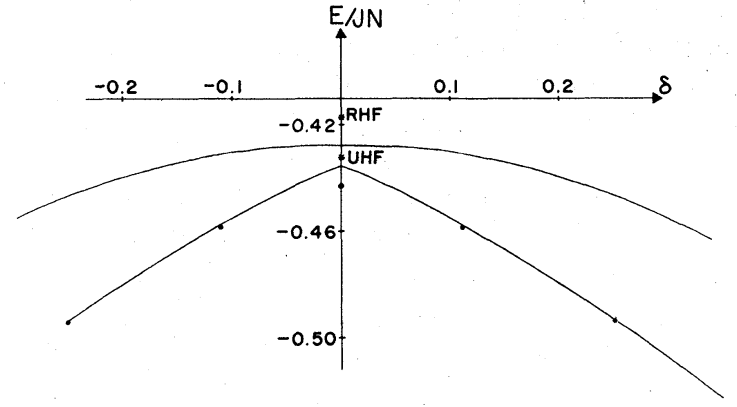

FIG. 2. Ground-state energy estimates for the $s=\frac{1}{2}$ linear Heisenberg model as a function of the alternation parameter $\delta$. The lower curve is for the present VB cluster ansatz, the upper curve is for a less accurate) nonsymmetric ansatz, and the points at $\delta=0, \pm 0.11$, \pm 0.25 are numerical estimates from Duffy and Barr (Ref. 20). The common RHF and UHF results for $\delta$ $=0$ are also shown.

where the matrix elements over $\overrightarrow{\mathrm{s}}_{1} \cdot \overrightarrow{\mathrm{s}}_{2}$ and $\overrightarrow{\mathrm{s}}_{2} \cdot \overrightarrow{\mathrm{s}}_{3}$ are given in Appendix A.

Numerical optimization with respect to $x, y$, and $z$ now yields the lower curve of Fig. 2, and the results of Table I. Also shown, in the upper curve of this figure, is the result for the two-site nonsymmetric (non-VB) cluster ansatz of a type discussed earlier ${ }^{19}$; this ansatz is treated in Appendix $B$. We see that it is only the VB ansatz. which predicts a cusp in this energy curve at $\delta=0$. Also in Fig. 2 we show the well-known ${ }^{5,6,13,14}$ restricted Hartree-Fock (RHF) and unrestricted Hartree- Fock (UHF) solutions (of the JordanWigner transformed $s=\frac{1}{2}$ Heisenberg model) both of which are surpassed in accuracy by the present VB ansatz. Although the RHF solution is ${ }^{5,6}$ as sociated with a bond alternation instability, the UHF solution apparently is not. ${ }^{14}$ Finally in Fig.

TABLE I. Selected results for the $s=\frac{1}{2}$ VB cluster ansatz (with a normalization such that $x=1.000$ ).

\begin{tabular}{cccc}
\hline \hline$\delta$ & $y$ & $z$ & $E_{\delta} / J N$ \\
\hline 0.00000 & 0.319 & 0.283 & -0.43576 \\
0.01010 & 0.311 & 0.266 & -0.43750 \\
0.02564 & 0.298 & 0.242 & -0.44030 \\
0.05263 & 0.278 & 0.207 & -0.44548 \\
0.09890 & 0.247 & 0.158 & -0.45524 \\
0.19760 & 0.194 & 0.090 & -0.47930 \\
0.29870 & 0.152 & 0.050 & -0.50753 \\
0.39860 & 0.118 & 0.028 & -0.53826 \\
0.50376 & 0.089 & 0.015 & -0.57228 \\
0.60000 & 0.066 & 0.008 & -0.60500 \\
0.69492 & 0.047 & 0.004 & -0.63829 \\
0.80180 & 0.028 & 0.001 & -0.67673 \\
0.90476 & 0.012 & 0.000 & -0.71451 \\
1.00000 & 0.0000 & 0.0000 & -0.75000 \\
\hline \hline
\end{tabular}


2 we also show the few points obtained by Duffy and $\mathrm{Barr}^{20}$ from their numerical extrapolation of finite chain energies, with their $\delta=0$ result being known to be very accurate. ${ }^{21}$ Their points do not seem to be inconsistent with a cusp at $\delta=0$. Further, the existence of a $\delta=0$ cusp can be argued to be consistent with their speculation ${ }^{20}$ that the zero-temperature specific-heat slope is nonzero only for $\delta=0$ : a ground-state cusp suggests, as $\delta$ is changed away from 0 , that the ground state drops away from the excited states, so that we expect an energy gap for spin-wave excitations, with this gap closing to zero just at $\delta=0$.

Next, we can compare results for the VB ansätze with the excitations truncated at two, four, and six sites. The LRO parameters for the two-, four-, and six-site cases are

$$
\rho=0.375,0.225,0.169,
$$

while the energies for the two-, four-, six-, and $\infty_{-}$site cases are

$E_{6} / J N=-0.3750,-0.4268,-0.4358,-0.4431$.

Comparison of the energy convergence to the changes in the LRO estimate is suggestive that $\rho$ remains nonzero even for very accurate $\mathrm{VB}$ ansätze.

Finally, calculations on finite even cyclic chains for $\delta=0$ may be consulted for some further evidence. First we note that $(+)$ and $(-)$ linear combinations of $|A\rangle$ and $|B\rangle$ Kekulé-like states should, for finite $N$, give states $|+\rangle$ and $|-\rangle$ with translational symmetries differing by $\pi$. Thus we expect the ground state of wave vector $k_{0}$ and the lowest lying state of wave vector $k_{0}+\pi$ to approach degeneracy at least as fast as $1 / N$. Checking this energy difference for chains of length $N=4,6,8$, 10 , and 12 we find $2.0000,1.3028,0.9515,0.7448$, and $0.6100 \mathrm{~J}$, respectively. Indeed, it is seen that this appears to extrapolate linearly in $1 / N$ to near the expected limit 0.00 .

\section{NUMERICAL RESULTS FOR $s=1$}

We employ the many-body cluster VB ansatz, with a wave function

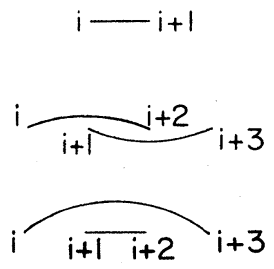

FIG. 3. Diagrams for the spin pairings occurring in (7.2).

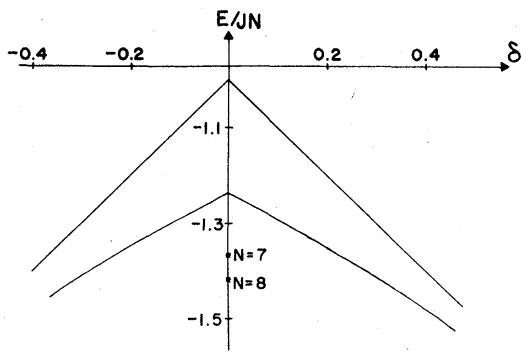

FIG. 4. Ground-state energy estimates for the $s=1$ linear Heisenberg model. The lower curve is for the present VB cluster ansatz, while the upper curve is for a single Kekule state. The energies obtained for finite cyclic chains of lengths $N=7$ and $N=8$ are also shown.

$$
\begin{aligned}
& |\Psi\rangle \equiv u_{s}\left(e^{T+}\right)|\Phi\rangle \\
& T^{+} \equiv \sum_{i=1}^{2 n}\left(T_{i i+1}^{+}+T_{i+1 i+2 i+3}^{+}\right),
\end{aligned}
$$

and

$$
\begin{aligned}
& T_{i i+1}^{+}\left|g_{i} g_{i+1}\right\rangle=x_{i}[i, i+1], \\
& T_{i i+1 i+2 i+3}^{+}\left|g_{i} g_{i+1} g_{i+2} g_{i+3}\right\rangle \\
& \quad=y_{i}[i, i+3][i+1, i+2]+z_{i}[i, i+2][i+1, i+3] .
\end{aligned}
$$

The Rumer-type diagrams associated with these excitations are displayed in Fig. 3. Again we have neglected four-site excitations giving rise to nearest neighbor spin-pairings as in $[i, i+1][i+2, i+3]$. Again too we may choose $x_{i}=y_{i}=z_{i}=0$ for either all even $i$ (if $\delta \leqslant 0$ ) or all odd $i$ (if $\delta \geqslant 0$ ), and we denote the remaining nonzero parameters simply by $x, y$, and $z$. The energy expression is of the form in (6.3) with the $\overrightarrow{\mathrm{s}}_{1} \cdot \overrightarrow{\mathrm{s}}_{2}$ and $\overrightarrow{\mathrm{s}}_{2} \cdot \overrightarrow{\mathrm{s}}_{3}$ matrix elements obtained in Appendix C.

Numerical optimization with respect to $x, y$, and $z$ yields the lower curve of Fig. 4, and the results of Table II. Also shown, in the upper curve of this figure, is the result of the simple Kekule states. Finally the $\delta=0$ points computed ${ }^{22}$ for cyclic chains of 7 and 8 sites are indicated; we note that odd and even cyclic chains for $s=\frac{1}{2}$ are

TABLE II. Selected results for the $s=1 \mathrm{VB}$ cluster ansatz (with $x=1.000$ ).

\begin{tabular}{cccc}
\hline \hline$\delta$ & $y$ & $z$ & $E_{\delta} / J N$ \\
\hline 0.000 & -0.353 & 0.420 & -1.23851 \\
0.050 & -0.334 & 0.391 & -1.26492 \\
0.100 & -0.315 & 0.364 & -1.29234 \\
0.150 & -0.296 & 0.337 & -1.32082 \\
0.200 & -0.276 & 0.311 & -1.35042 \\
0.300 & -0.237 & 0.261 & -1.43132 \\
0.400 & -0.199 & 0.214 & -1.48109 \\
\hline \hline
\end{tabular}


observed to yield upper and lower bounds to the exact infinite chain energy per site, and such a speculation seems to us also to be consistent with the available $s=1$ data. $^{22}$ The value we obtain for the LRO from our present calculation is $\rho \cong 0.52$. Because of the seemingly rather large energy error and the rather large change in $\rho$ from that of the Kekule state, this calculation is not so supportive of the $\rho \neq 0$ prediction for $s=1$, as were the calculations for the $s=\frac{1}{2}$ case.

\section{DISCUSSION}

Our predictions of ground-state dimerizing instabilities are based upon the known global-singlet character of the ground state along with an assumption concerning local-singlet character, this assumption being that singlet spin pairing (or coupling) occurs almost totally amongst nearer-neighbor sites (as is typical in conventional VB treatments of molecular electronic structure, where our "sites" are identified as $s=\frac{1}{2}$ electrons). The combination of global- and local-singlet symmetry characteristics then implies an asymptotic degeneracy at $\delta=0$, and deviation away from $\delta=0$ yields a linear energy response, much as in the conventional Jahn-Teller effect.

The arguments we have presented concerning ground state dimerizing instabilities have required several conditions of the system, including: (a) covalent structures only are of importance; (b) exchange interactions are one dimensional; (c) the Heisenberg model is isotropic with no magnetic field; and (d) the temperature is $0^{\circ} \mathrm{K}$. The lifting of any of these conditions would in general cause our local-singlet assumption not to preclude the admixture of type- $C$ VB structures and hence spoil our argument leading to LRO and dimerization. Another type of condition for LRO is, naturally, that the chains be very long. Of course in any real system each of these conditions is generally violated to some small degree. However, if the violation is sufficiently weak, all that occurs is that the curvature in our energy curves at $\delta=0$, instead of being infinite, is now finite but very large in magnitude. If this magnitude exceeds that of the relevant (zero-temperature) elastic constant, dimerization still occurs, and at finite temperatures spin-Peierls phase transitions can occur. ${ }^{4-6}$ On the other hand, there are some assumptions in the preceeding sections which do not seem to be necessary to our LRO conclusion. Thus, for instance, we could include next-nearest neighbor or biquadratic exchange in our Heisenberg Hamiltonians.

It can be argued that our view of the dimerizing instability in Heisenberg models is more reliable than those previously given. There are two major points in the argument: (i) our energies are more accurate (i.e., lower) than both the ${ }^{5,6}$ RHF solutions and the $\mathrm{e}^{13,14}$ UHF solutions; and (ii) a physically reasonable local-singlet symmetry assumption indicates the dimerizing instability persists for even very accurate wave functions. In addition, our predictions apply to a wider variety of linear isotropic Heisenberg models. The prediction for the $s=\frac{1}{2}$ case seems most reliable though, because the main assumption of Sec. III or IV seems consistent with chemical VB ideas, and because our calculations of Sec. VI more strongly support $\rho \neq 0$. Further there are some rather special models to which our wave function ansätze and local symmetry arguments apply exactly. An example in point is the special ${ }^{23}$ Heisenberg model.

$$
\begin{aligned}
H=J \sum_{i}^{\text {odd }}[ & (1+\delta) \overrightarrow{\mathrm{s}}_{i} \cdot \overrightarrow{\mathrm{s}}_{i+1}+(1-\delta) \overrightarrow{\mathrm{s}}_{i-1} \cdot \overrightarrow{\mathrm{s}}_{i}+\frac{1}{2} \overrightarrow{\mathrm{s}}_{i} \cdot \overrightarrow{\mathrm{s}}_{i+2} \\
+ & \left.+\frac{1}{2} \overrightarrow{\mathrm{s}}_{i-1} \cdot \overrightarrow{\mathrm{s}}_{i+1}\right]
\end{aligned}
$$

which at $\delta=0$ have the Kekule states of (2.2) as degenerate eigenkets; for the $s=\frac{1}{2}$ case the associated eigenvalue is known to be that for the ground state.

Finaily, it seems that the VB calculations of Secs. VI and VII are the first many-body VB calculations. Especially in view of the physical appeal and of the qualitative differences between VB wave functions and the usual (Hartree-Fock and Green's function) treatments further applications should be made.

\section{APPENDIX A: VB CLUSTER ANSATZ FOR $s=\frac{1}{2}$ CHAINS}

In the appendices here we use diagrams with a single vertical line (rather than a single point as in a Rumer or superposition diagram), and we stack up various diagrammatic components with top-to-bottom diagram ordering corresponding to left-to-right operator ordering. Further, we use the matrix-element evaluation nomenclature and techniques already described ${ }^{17,18}$ elsewhere.

Before considering the Hamiltonian matrix elements for the wave function of (6.1) we wish to evaluate the "residual overlap ratios" via the socalled strongly linked expansion formulas ${ }^{17}$
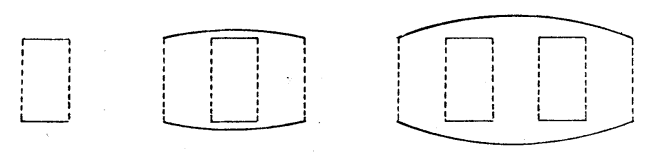

FIG. 5. Closed (strongly linked) diagrams arising in evaluating the overlap elements for the $s=\frac{1}{2} \mathrm{VB}$ ansatz. 

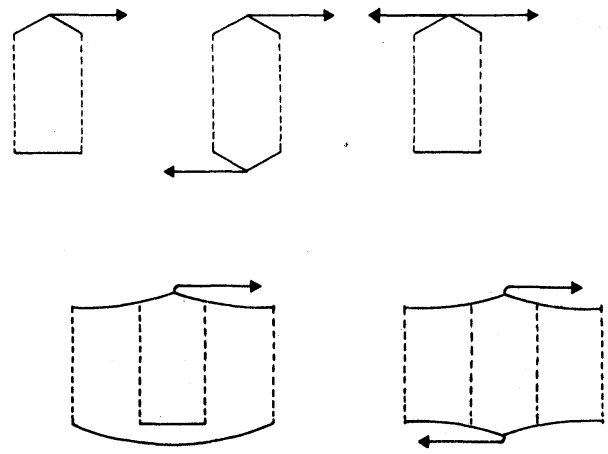

FIG. 6. Strongly linked dangling diagrams arising for the overlaps. All remaining strongly linked dangling diagrams are obtained from those shown by inversions or reflections. Hence, there are $4,2,2,4$, and 2 diagrams of the respective types shown here.

$$
\begin{aligned}
& f_{12} \equiv \frac{\langle\Psi \mid \Psi\rangle}{\langle\Psi \mid \Psi\rangle_{(12)}}=\sum_{m}^{\text {even }} \mathcal{L}^{12 \cdots m}\left(1_{1}\right) \frac{m}{f^{m / 2-1}}, \\
& f \equiv \frac{\langle\Psi \mid \Psi\rangle_{(12)}}{\langle\Psi \mid \Psi\rangle_{(1234)}}=\sum_{m}^{\text {even }} \mathscr{L}^{(12) 34 \cdots m+2}\left(1_{3}\right) \frac{1}{f^{m / 2-1}},
\end{aligned}
$$

where a subscript $(12 \ldots j)$ appended to an overlap matrix element indicates sites $12 \ldots j$ are deleted from the wave functions so subscripted, and the script- $\mathcal{L}$ terms are "strongly linked sums," which involve sums over linked few-site diagrams. Of these linked diagrams there are "closed" ones, given in Fig. 5, and "dangling" ones, given in Fig. 6. Arrows have not been included on the pairing lines, because our infinite linear case is an even alternate (bipartite) graph for which we can entirely avoid phases. ${ }^{12}$ The closed diagrams of Fig. 5 are easily evaluated, using the techniques of Sec. IV of the preceeding paper, to yield $2 x^{2}, 4 y^{2}$, and $8 z^{2}$. Now the first of the dang-

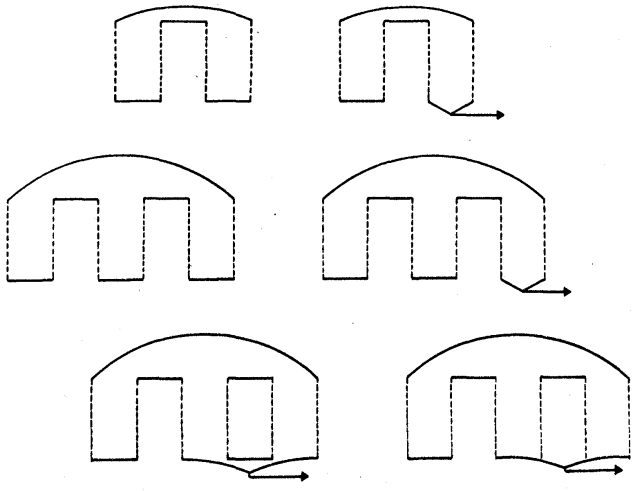

FIG. 7. Higher-order diagrams arising in the expansion of the first diagram in Fig. 6. Although linked, these diagrams are no longer strongly linked, since more than one excitation operator is here required on the ket side of the corresponding matrix elements.

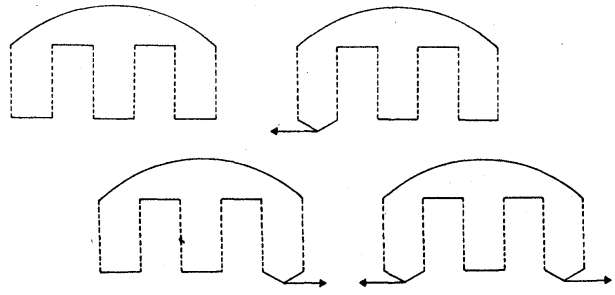

FIG. 8. Higher-order diagrams arising in the expansion of the third diagram in Fig. 6 .

ling diagrams of Fig. 6 can be expanded ${ }^{18}$ as $1 / f$ times the sum of the first two diagrams plus $1 / f^{2}$ times the sum of the last four diagrams, all in Fig. 7. The second dangling diagram of Fig. 6 is expanded similarly, with the lower left bar in the diagrams of Fig. 7 replaced by the left-pointing arrow structure as found on the bottom of this second dangling diagram. The third dangling diagram of Fig. 6 is expanded as $1 / f^{4}$ times the sum of the diagrams in Fig. 8. Next, the fourth dangling diagram is expanded in terms of those in Fig. 9, with those for the fifth diagram being very similar. Now denoting the values of the first, third, and fourth dangling diagrams, of Fig. 6 , by $x \xi, x \eta$, and $y \zeta$, we see that these expansions just described above lead to recurrence relations

$$
\begin{aligned}
& \xi=(2 x y+y \xi) \frac{1}{f}+\left(2 z x^{2}+z x \xi+4 z y+z \zeta\right) \frac{1}{f^{2}} \\
& \eta=\left(2 x^{2}+2 x \xi+\frac{1}{2} \xi^{2}\right) \frac{z}{f^{2}}, \\
& \zeta=(4 x z+2 z \xi) \frac{1}{f} .
\end{aligned}
$$

From these we find,

$$
\xi=\left(\frac{2 x y}{f}+\left(x^{2}+2 y\right) \frac{2 z}{f^{2}}+\frac{4 x z^{2}}{f^{3}}\right)\left(1-\frac{y}{f}-\frac{x z}{f^{2}}-\frac{2 z^{2}}{f^{3}}\right)^{-1}
$$

which in turn determines $\eta$ and $\zeta$ (once $x, y, z$, and $f$ are known). Further because of the similarity of the second and fifth dangling diagrams of Fig. 6 to the first and fourth ones, we see that they take values $\frac{1}{2} \xi^{2}$ and $\frac{1}{2} \zeta^{2}$. Then, the nonzero strongly linked sums are
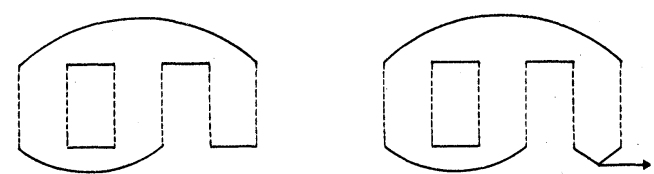

FIG. 9. Higher-order diagrams arising from the fourth of Fig. 6 . 


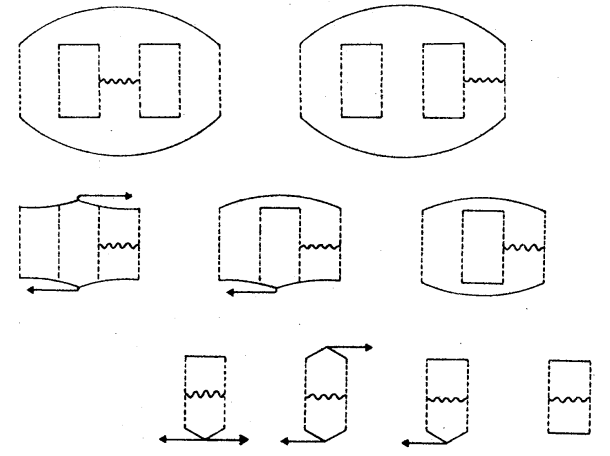

FIG. 10. Various strongly linked diagrams with an interaction line disposed as appropriate for the first equation of (A 5). The symmetry numbers for these diagrams are $1,4,2,4,2,8,2$, and 1 , respectively.

$$
\begin{aligned}
& \mathcal{L}^{(12) 34}\left(1_{3}\right)=2 x^{2}+2 x \xi, \\
& \mathcal{L}^{(12) 3456}\left(1_{3}\right)=4 y^{2}+2 y \zeta, \\
& \mathscr{L}^{(12) 345678}\left(1_{3}\right)=8 z^{2}, \\
& \mathcal{L}^{12}\left(1_{1}\right)=2 x^{2}+4 x \xi=\xi^{2}+2 x \eta, \\
& \mathcal{L}^{1234}\left(1_{1}\right)=4 y^{2}+4 y \zeta+\zeta^{2}, \\
& \mathcal{L}^{123456}\left(1_{1}\right)=8 z^{2} .
\end{aligned}
$$

Hence, the residual overlap ratios of (A1) can be determined self-consistently in terms of themselves.

Having found the $f$ 's we now consider the Hamiltonian matrix elements, which are developed in terms of similar strongly linked expansions

$$
\begin{aligned}
\frac{\left\langle\Psi\left|\overrightarrow{\mathrm{S}}_{1} \cdot \overrightarrow{\mathrm{s}}_{2}\right| \Psi\right\rangle}{\langle\Psi \mid \Psi\rangle}=\frac{1}{f_{12}}\left(\mathcal{L}^{12}\left(\overrightarrow{\mathrm{s}}_{1} \cdot \overrightarrow{\mathrm{s}}_{2}\right)+\mathcal{L}^{1234}\left(\overrightarrow{\mathrm{s}}_{1} \cdot \overrightarrow{\mathrm{s}}_{2}+\overrightarrow{\mathrm{s}}_{3} \cdot \overrightarrow{\mathrm{s}}_{4}\right) \frac{1}{f}\right. \\
\left.+\mathcal{L}^{123456}\left(\overrightarrow{\mathrm{s}}_{1} \cdot \overrightarrow{\mathrm{s}}_{2}+\overrightarrow{\mathrm{s}}_{3} \cdot \overrightarrow{\mathrm{s}}_{4}+\overrightarrow{\mathrm{S}}_{5} \cdot \overrightarrow{\mathrm{s}}_{6}\right) \frac{1}{f^{2}}\right),
\end{aligned}
$$

$$
\begin{aligned}
\frac{\left\langle\Psi\left|\overrightarrow{\mathrm{s}}_{2} \cdot \overrightarrow{\mathrm{s}}_{3}\right| \Psi\right\rangle}{\langle\Psi \mid \Psi\rangle}=\frac{1}{f_{12}}\left(\mathcal{L}^{1234}\left(\overrightarrow{\mathrm{s}}_{2} \cdot \overrightarrow{\mathrm{s}}_{3}\right) \frac{1}{f}\right. \\
\left.+\mathscr{L}^{123456}\left(\overrightarrow{\mathrm{s}}_{2} \cdot \overrightarrow{\mathrm{s}}_{3}+\overrightarrow{\mathrm{s}}_{4} \cdot \overrightarrow{\mathrm{s}}_{5}\right) \frac{1}{f^{2}}\right)
\end{aligned}
$$

where we have noted the translational symmetry of $|\Psi\rangle$ with the bonds between sites $2 i-1$ and $2 i$ identified as the "strong" bonds, for which nonzero variational parameters appear in our VB ansatz.
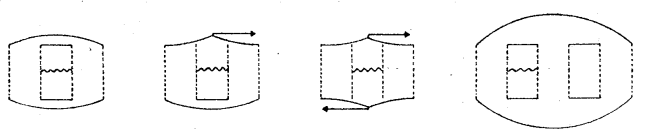

FIG. 11. Various strongly linked diagrams associated with the second line of (A5). Their respective symmetry numbers are 1,42 , and 2 .
The diagrams appearing in the first and second equations of (A5) are given in Figs. 10 and 11, and lead to the results

$$
\begin{aligned}
& \mathcal{L}^{12}\left(\overrightarrow{\mathrm{s}}_{1} \cdot \overrightarrow{\mathrm{s}}_{2}\right)=-\frac{3}{4}\left(2 x^{2}+4 x \xi+\xi^{2}+2 x \eta\right), \\
& \mathcal{L}^{1234}\left(\overrightarrow{\mathrm{s}}_{1} \cdot \overrightarrow{\mathrm{s}}_{2}+\overrightarrow{\mathrm{S}}_{3} \cdot \overrightarrow{\mathrm{s}}_{4}\right)=0, \\
& \mathcal{L}^{123456}\left(\overrightarrow{\mathrm{s}}_{12} \cdot \overrightarrow{\mathrm{s}}_{2}+\overrightarrow{\mathrm{s}}_{3} \cdot \overrightarrow{\mathrm{s}}_{4}+\overrightarrow{\mathrm{s}}_{5} \cdot \overrightarrow{\mathrm{s}}_{6}\right)=0, \\
& \mathcal{L}^{1234}\left(\overrightarrow{\mathrm{s}}_{2} \cdot \overrightarrow{\mathrm{s}}_{3}\right)=-\frac{3}{4}\left(y^{2}+4 y \zeta+\zeta^{2}\right), \\
& \mathcal{L}^{123456}\left(\overrightarrow{\mathrm{s}}_{2} \cdot \overrightarrow{\mathrm{s}}_{3}+\overrightarrow{\mathrm{s}}_{4} \cdot \overrightarrow{\mathrm{s}}_{5}\right)=-\frac{3}{4} \times 16 z^{2},
\end{aligned}
$$

which then determine the Hamiltonian matrix elements.

\section{APPENDIX B: NONSYMMETRIC ANSATZ FOR $s=\frac{1}{2}$}

The nonsymmetric localized-site cluster ansatz developed earlier may also be appled to the alternating Heisenberg chain. This wave-function ansatz is (most simply)

$$
\begin{aligned}
& |\Psi\rangle \equiv \mathcal{u}\left(e^{T^{+}}\right)|\Phi\rangle \\
& |\Phi\rangle \equiv \alpha \beta \alpha \beta \ldots \alpha \beta \\
& T^{+} \equiv x \sum_{i=1}^{n}(2 i-1,2 i)+y \sum_{i=1}^{n}(2 i, 2 i+1),
\end{aligned}
$$

where $(i j)$ is a transposition interchanging spin indices $i$ and $j$. The nonalternating case (with $x=y$ ) has already been considered ${ }^{19}$ and we can follow that development very closely. In evaluating the residual overlap ratios only a single type of linked diagram occurs and is associated with the values $x^{2}$ or $y^{2}$, depending on the bond involved Our first residual overlap ratios are given as

$$
f_{2}=1+x^{2} / f_{(2) 1}+y^{2} / f_{(2) 3}, \quad f_{3}=1+y^{2} / f_{(3) 2}+x^{2} / f_{(3) 4} .
$$

As usual for infinite open chains the residual overlap ratios $f_{(i) i-1}$ involving the deletion of sites on the end of the chain achieve an independence of the chain length, and we obtain

$$
\begin{aligned}
& f_{x} \equiv f_{(2) 3}=f_{(3) 2}=1+x^{2} / f_{y}, \\
& f_{y} \equiv f_{(2) 1}=f_{(3) 4}=1+y^{2} / f_{x} .
\end{aligned}
$$

Combining these two equations and solving, we find

$$
f_{x}=\frac{1+y^{2}-x^{2}}{2}+\left[\left(\frac{1+y^{2}-x^{2}}{2}\right)^{2}+x^{2}\right]^{1 / 2} \text {. }
$$

For matrix elements over $2 \overrightarrow{\mathrm{s}}_{i} \cdot \overrightarrow{\mathrm{s}}_{i+1}+\frac{1}{2}$ exactly the same type of diagrams arise as did $^{19}$ in the uniform case, so that, taking care of the effects of alternation, we presently find

$\left\langle\Psi\left|\overrightarrow{\mathbf{s}}_{1} \cdot \overrightarrow{\mathbf{s}}_{2}\right| \Psi\right\rangle /\langle\Psi \mid \Psi\rangle=\left(1 / 2 f_{1} f_{y}\right)\left(x+y^{2} / f_{x}\right)-\frac{1}{2}$,

$\left\langle\Psi\left|\overrightarrow{\mathbf{s}}_{2} \cdot \overrightarrow{\mathbf{s}}_{3}\right| \Psi\right\rangle /\langle\Psi \mid \Psi\rangle=\left(1 / 2 f_{2} f_{x}\right)\left(y+x^{2} / f_{y}\right)-\frac{1}{2}$ 


\section{APPENDIX C: VB CLUSTER ANSATZ FOR $s=1$ CHAINS}

We consider the ansatz of (7.1) with two- and four-site excitations. The treatment uses the same techniques as for the $s=\frac{1}{2}$ ansatz of Appen$\operatorname{dix} A$. In evaluating the residual overlap ratios the various closed and dangling diagrams which arise are shown in Figs. 12 and 13. The closed diagrams take values $3 x^{2}, 9 y^{2}, 3 y z$, and $9 z^{2}$. Letting the first dangling diagram of Fig. 13 have the value $x \xi$, and expanding via the type of procedure indicated in Appendix A, we obtain

$$
x \xi=\left(3 x^{2} y+x y \xi+3 x^{2} z+x z \xi\right) / f^{2},
$$

so that

$$
\xi=3 x(y+z)\left(f^{2}-y-z\right)^{-1} .
$$

The second dangling diagram of Fig. 13 is simply $\frac{1}{3} \xi^{2}$. Consequently, the residual overlap ratios of interest are given as

$f_{12} \equiv \frac{\langle\Psi \mid \Psi\rangle}{\langle\Psi \mid \Psi\rangle_{(12)}}=\left(3 x^{2}+4 x \xi+\frac{2}{3} \xi^{2}\right) 2+\left(9 y^{2}+6 y z+9 z^{2}\right) \frac{4}{f}$,

$f \equiv \frac{\langle\Psi \mid \Psi\rangle_{(12)}}{\langle\Psi \mid \Psi\rangle_{(1234)}}=\left(3 x^{2}+2 x \xi\right)+\left(9 y^{2}+6 y z+9 z^{2}\right) \frac{1}{f^{2}}$.

Next, for the energy expression we find the diagrams of Figs. 14 and 15 involved in the strongand weak-bond matrix elements. Then,

$$
\begin{aligned}
& \frac{\left\langle\Psi\left|\overrightarrow{\mathrm{s}}_{1} \cdot \overrightarrow{\mathrm{s}}_{2}\right| \Psi\right\rangle}{\langle\Psi \mid \Psi\rangle}=\frac{-2}{f_{12}}\left(3 x^{2}+4 x \xi+\frac{2}{3} \xi^{2}+\frac{12 y z}{f}\right), \\
& \begin{aligned}
\frac{\left\langle\Psi\left|\overrightarrow{\mathrm{s}}_{2} \cdot \overrightarrow{\mathrm{s}}_{3}\right| \Psi\right\rangle}{\langle\Psi \mid \Psi\rangle}=\frac{2}{f_{12} f} & \left(6 x^{2} y-6 x^{2} z-6 y z-9 z^{2}+4 x y \xi\right. \\
& \left.+\frac{2}{3} y \xi^{2}-4 x z \xi-\frac{2}{3} z \xi^{2}\right) .
\end{aligned}
\end{aligned}
$$

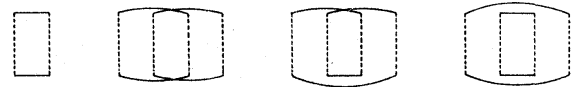

FIG. 12. Closed diagrams for the overlaps of the $s$ $=1 \mathrm{VB}$ ansatz.

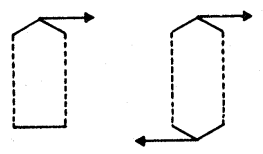

FIG. 13. Strongly linked dangling diagrams (with symmetry numbers 4 and 2) for the overlaps of the $s$ $=1 \mathrm{VB}$ ansatz.

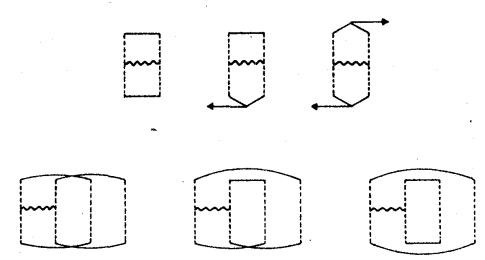

FIG. 14. Strongly.linked diagrams with an interaction line for the strong bond, as associated with the first equation of (C4). Their respective symmetry numbers are $1,4,2,2,4$, and 2 .

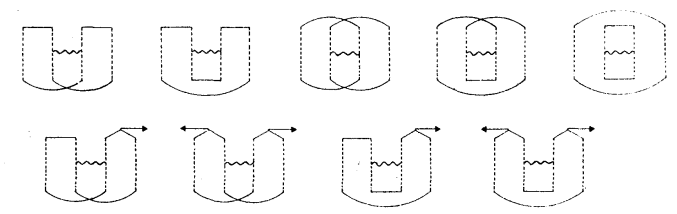

FIG. 15. Strongly linked diagrams (with symmetry numbers $2,2,1,2,4,2,4$, and 2) associated with the second equation of (C4).
*Present address: Department of Chemistry, Rice University, Houston, TX 77001.

$\dagger$ Present address: Depto. de Fisica Teorica, Universidad de Barcelona, Av. Generalismo 647, Barcelona, 14 Spain.

${ }^{1}$ R. E. Peierls, Quantum Theory of Solids (Oxford U.P., London, 1955), p. 108.

${ }^{2}$ H. Frohlich, Proc. R. Soc. A 223, 296 (1954); C. G. Kuper, ibid. 227, 214 (1955); P. A. Lee, T. M. Rice, and P. W. Anderson, Solid State Commun. 14, 703 (1974).

${ }^{3}$ H. M. McConnell and R. M. Lynden-Bell, J. Chem. Phys. 36, 2393 (1962); D. D. Thomas, H. Keller, and H. M. McConnell, ibid. 39, 2321 (1963).

${ }^{4}$ D. B. Chesnut, J. Chem. Phys. 45, 4677 (1966); K. A. Penson, A. Holz, and K. H. Bennemann, ibid. 65, 5024 (1976).

${ }^{5}$ G. Beni, J. Chem. Phys. 58, 3200 (1973); E. Pytte, Phys. Rev. B 10, 4637 (1974).

${ }^{6}$ J. W. Bray, H. R. Hart, Jr., L. V. Interrante, I. S.
Jacobs, J. S. Kasper, G. D. Watkins, S. H. Wee, and J. C. Bonner, Phys. Rev. Lett. 35, 744 (1975); I. S. Jacobs, J. W. Bray, H. R. Hart, Jr., L. V. Interrante, J. S. Kasper, G. D. Watkins, D. E. Prober, and J. C. Bonner, Phys. Rev. B 14, 3036 (1976); L. S. Smith, E. Ehrenfreund, A. J. Heeger, L. V. Interrante, J. W. Bray, H. R. Hart, Jr., and I. S. Jacobs, Solid State Commun. 19, 377 (1976).

${ }^{7}$ R. Comes, M. Lambert, H. Launois, and H. R. Zeller, Phys. Rev. B 8,571 (1973); B. Renker, H. Rietschel, L. Pintschouvius, N. Glaser, P. Bruesch, D. Kuse, and M. J. Rice, Phys. Rev. Lett. 30, 1144 (1973); H. Niedoba, H. Launois, D. Brinkman, R. Brugger, and H. R. Zeller, Phys. Status Solidi B 58, 309 (1973); F. Denoyer, R. Comes, A. F. Garito, and A. J. Heeger, Phys. Rev. Lett. 35, 445 (1975); H. A. Mook and C. A. Watson, Jr., ibid. 36, 801 (1976); S. Kagoshima, H. Anzoi, K. Kajimura, and T. Ishiguro, J. Phys. Soc. Jpn. 41, 2061 (1976).

${ }^{8} \mathrm{See}$, for instance, the articles and further references, 
in One-Dimensional Conductors, edited by H. G. Schuster (Springer-Verlag, Berlin, 1975).

${ }^{9}$ See, for instance, H. Labhart, J. Chem. Phys. 27, 963 (1957); Y. Ooshika, J. Phys. Soc. Jpn. 12, 1238, 1246 (1957); H. C. Longuet-Higgins and L. Salem, Proc. R. Soc. A 251, 172 (1959); G. Binsch, E. Heilbronner, and J. N. Murrell, Mol. Phys. 11, 305 (1966); J. Paldus and J. Cizek, Phys. Rev. A 2 , 2268 (1970); A. A. Ovchinnikov, I. I. Ukranskii, and G. V. Kventsel, Usp. Fiz. Nauk 108, 81 (1972) [Sov. Phys.-Usp. 15, 575 (1973)].

${ }^{10} \mathrm{C}$. A. Coulson and W. T. Dixon, Tetrahedron 17, 215 (1962).

${ }^{11}$ Although this argument is rather straightforward, and similar to that of Coulson and Dixon (Ref. 10), it is occusionally asserted that such predictions are contrary to simple VB or resonance theory. The basis of such assertions is apparently found in the often given and successful resonance theory rationalization of the benzene structure.

${ }^{12}$ D. J. Klein, preceding paper, Phys. Rev. B 19, 870, (1979).

${ }^{13}$ T. W. Ruijgrok and S. Rodriquez, Phys. Rev. 119 , 596 (1960); Z. G. Soos, J. Chem. Phys. 43, 1121 (1966).
${ }^{14}$ D. B. Abraham and A. D. Mc Lachlan, Mol. Phys. 12, 319 (1967);

${ }^{15}$ E. H. Lieb and D. C. Mattis, J. Math. Phys. 3,749 (1962).

${ }^{16}$ In more detail this follows because an overlap $\langle A \mid B\rangle$, between two normalized VB structures of types $A$ and $B$, must involve a large island with member sites from all around the cycle, so that many powers of 2 will be lost in forming this large island instead of many small ones, as in $\langle A \mid A\rangle$ or $\langle B \mid B\rangle$. If the average distance between sites in the large island of $\langle A \mid B\rangle$ is $d$, then $\langle A \mid B\rangle \leqslant(2 s+1)^{-n d+1}$. The noninteracting feature follows again since $I$ can introduce very few new islands.

${ }^{17}$ D. J. Klein, Int. J. Quantum Chem. 12, 255 (1977).

${ }^{18}$ M. A. Garcia-Bach and D. J. Klein, Int. J. Quantum Chem. 12, 273 (1977)

${ }^{19}$ D. J. Klein, Mol. Phys. 31, 811 (1976).

${ }^{20}$ W. Duffy, Jr., and K. P. Barr, Phys. Rev. 165, 647 (1968).

${ }^{21}$ L. Hulthén, Ark. Mat. Astron. Fys. A 26, 1 (1938). ${ }^{22} \mathrm{~T}$. de Neef, Phys. Rev. B 13, 4141 (1976).

${ }^{23}$ C. K. Majumdar, J. Phys. C 3 , 911 (1970); D. J. Klein and T. L. Welsher (unpublished). 